\title{
The fishmouth phenomenon in retinal detachment: old concepts revisited
}

\author{
COLIN H. BIRCHALL \\ From the Department of Ophthalmology, Aberdeen Royal Infirmary and Woodend Hospital, Aberdeen
}

SUMMARY The 'fishmouth phenomenon' seen in some previously treated retinal detachments is associated with large horseshoe tears and scleral buckling techniques. A method of treating patients with this complication is described in which scleral buckling with implant or explant is not used. The technique utilises intravitreal air tamponade with cryopexy and gives good results. The incidence of patients developing the fishmouth phenomenon as a complication of primary simple retinal detachment surgery is much lower when an intravitreal air technique is used than the expected incidence following a primary scleral buckling operation.

The objective of this paper is to describe the management of difficult large posterior horseshoe-tear retinal detachments which develop the aptly named 'fishmouth phenomenon', in which a second surgical procedure is required following a failure of scleral buckling with scleral silicone implant or explant and/or encircling silicone tape.

The prognosis for reattachment of the retina in routine retinal detachment surgery for primary rhegmatogenous detachments, by different surgical techniques, is now well documented. The results from different series indicate reattachment in 90 to $95 \%$ of patients (Kreiger et al., 1971; Chignell, 1977; and Curtin, 1976). The reduced chance of a successful first surgical procedure in more complicated detachments, for example, detachments due to macular holes (Leaver and Cleary, 1975) and in secondary detachments is also recognised.

Results of operations for detachment where the primary process of detachment involves retinal lesions with large posterior tears suggest that the larger the tear the worse the prognosis (Lincoff et al., 1977). At the worst a giant tear may extend through almost $360^{\circ}$ and is virtually unmanageable with present surgical techniques. Difficulties are encountered in routine scleral buckling procedures with large horseshoe tears when the buckle causes the operculum of the tear to elevate as the rolled edges of the tear come closer together. This compromising effect is greater with circumferential buckles than with radial buckles. If further tightening of an encircling tape is attempted at this time, it

Address for reprints: Mr C. H. Birchall, 37 Forest Road, Aberdeen, Scotland AB2 4BY serves only to elevate the operculum further, hence the fishmouth phenomenon (Figs. 1 and 2).

Recent descriptions of the geometrical considerations of the sequelae of scleral buckling (Pruett, 1977) correctly and succinctly conclude that 3 factors contribute to the pathogenesis of the fishmouth phenomenon. These factors are: (1) the relative length of the buckle (relative to the circle of the eye that is being indented); (2) the height of the buckle, which in turn is related to the tension of the encircling tape and the depth of cross-section of the implant or explant; and (3) the shape of the edge of the implant or explant used.

Thus the fishmouth formation is associated with large horseshoe tears and scleral buckling techniques, but it has not been observed in the past 4 years by this author when such tears have been treated surgically without scleral implanting or explanting. It seems logical, therefore, to treat patients with large horseshoe tears, if they require reoperation, without any scleral implant or explant, and results from this series indicate that this can be satisfactorily performed.

\section{Method}

All the patients had been previously treated with conventional scleral buckling procedures with silicone or Silastic explants, cryopexy (1 with diathermy), and both with and without drainage of subretinal fluid. The distribution of tears is evaluated with reference to the 4 o'clock and 8 o'clock meridia, 2 of the patients having inferior tears with reference to these meridia, all the rest having superior tears (Fig. 3). The significance of these meridia in relation 


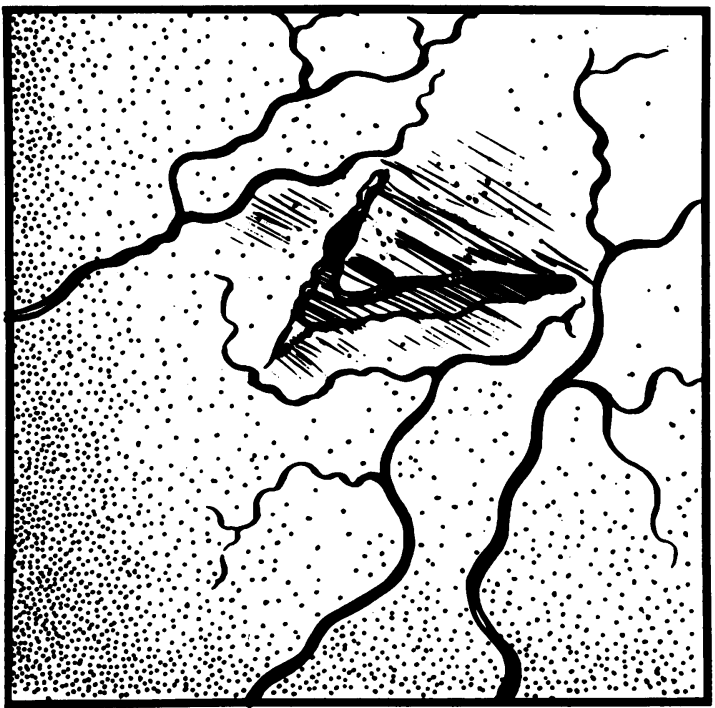

Fig. 1 Large horsehoe tear in posterior retina showing operculum relatively close to the retinal plane

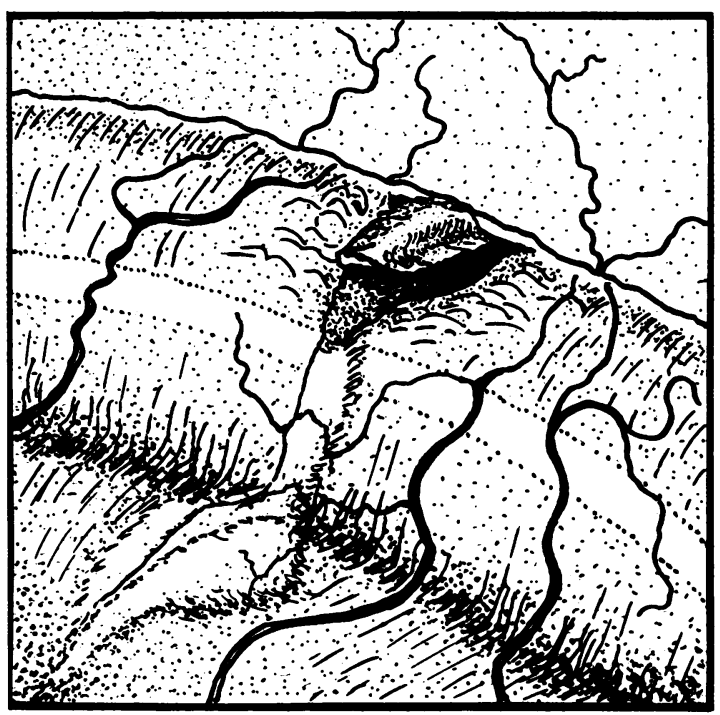

Fig. 2 Same large horseshoe tear as in Fig. 1 after circumferential scleral buckle has caused reduction in width of tear with elevation of operculum and increase in radial retinal fold

to the use of intravitreal air to float the retina up against the cryocoagulated choroid and scleral bed in detachment surgery has been indicated in a previous paper (Chawla and Birchall, 1973). After routine evaluation patients undergo surgery, and all encircling tapes and explants are removed at the outset. Areas of previous cryopexy are noted, and

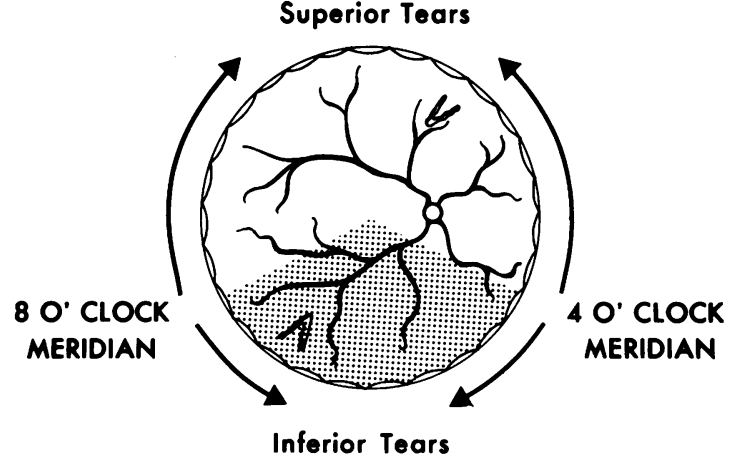

Fig. 3 In primary detachment procedures all patients with tears in the inferior shaded area have either an augmented intravitreal air technique with scleral buckling or straightforward scleral buckling. With tears in the superior nonshaded area intravitreal air is used without scleral buckling

any areas in the vicinity of the tear(s) that have not been frozen are treated together with the area immediately over the tear(s) and a wide surrounding area equivalent to 1 ring of cryo applications external to any previous cryopexy. Subretinal fluid is released by scleral scratch incision and choroidal puncture at a point distant from the tear(s), and in an area of high detachment inferiorly if possible, where any dependent fluid collects. Care is taken not to drain fluid in the equatorial meridian.

With the eye potentially hypotonic the intraocular tension is maintained by means of pressure externally from a cotton-tip applicator, and a pars plana injection of sterile air is made into the vitreous cavity. The intraocular tension is maintained and the injection is discontinued while the eye is still soft.

A no. 40 silicone tape is placed beneath the insertions of the rectus muscles and the ends are secured with a tantalum clip. This tape is then slowly moved posteriorly until it reaches the equator and it is secured there in all 4 quadrants with Dacron sutures. When any horseshoe tear(s) is straddling the equator the no. 40 tape is secured in that quadrant posterior to the tear. The tape does not indent over any tear(s), and no implant or explant is used to buckle the sclera.

After the operation the patients are positioned in bed, and when possible in bedside chairs, so that the intravitreal air covers the site of the tear(s) internally. In the 2 patients with inferior tears positioning the air bubbles in the vitreous to cover the tear(s) required them to lie flat, without pillows, with the foot of the bed slightly raised. This position was maintained for 48 hours in 1 patient and 72 hours in the second. Depending on the volume of intravitreal air used the air bubbles re-absorb in 5 to 9 days. 
VISUAL ACUITY OF PATIENTS TREATED

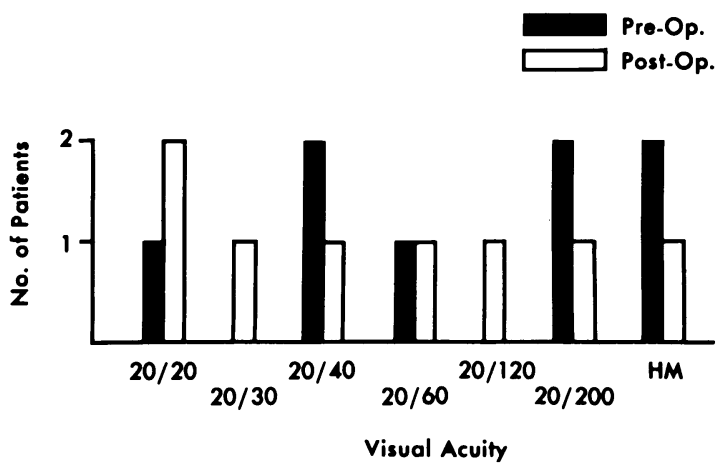

Fig. 4 Preoperative and postoperative visual acuities of 8 patients with secondary fishmouth phenomenon detachments treated with intravitreal air technique

\section{Results}

In this series 8 patients were treated in the above manner. Two of the patients had inferior tears as defined. Four patients had macular detachments of unknown duration, but none had any clinically appreciable vitreous retraction. All the patients had anatomical reattachment of the retina with 1 operation by the method described above. Complications were remarkably few despite the fact that the patients had previously undergone detachment surgery with scleral buckles. One patient suffered an operative complication. This was a small subretinal haemorrhage from the site of choroidal puncture, but postural treatment prevented it from reaching the posterior pole.

The best postoperative visual acuity of the 4 patients with macular detachments was $20 / 120 \mathrm{pt}$ (6/36 pt metric), the worst was hand movements at $1 \mathrm{~m}$. In the patients with attached maculae at the time of operation the final visual acuities range between 20/20 (6/6 metric) and 20/40 (6/12 metric). Follow-up time was 6 months to 2 years 3 months (Fig. 4).

\section{Discussion}

Many methods of surgical correction for redetachments due to fishmouth formation have been described (Lincoff et al., 1965; Schepens, 1965; Kreiger, 1971; Shea et al., 1972; Pruett 1977) and all include some type of scleral implant or explant. Lincoff et al. (1977) in the later part of their series, used gas or air alone in some cases of very large horseshoe tears and they also combined the use of these agents with radial buckling in other cases.

The method described above does not use any buckling implant or explant, and the no. 40 silicone tape is carefully positioned not to indent over the retinal tear(s). The series is small and the results are not statistically significant. This in itself poses some questions.

In a recent article in which the criteria for the production of fishmouthing were examined the expected incidence of the phenomenon was $10 \%$ of a series of 560 patients with primary rhegmatogenous detachment (Pruett, 1977). Of the present author's last 90 patients examined preoperatively for primary rhegmatogenous detachment 8 patients fulfilled criteria for the subsequent fishmouthing of their retinal tears. However, in practice after detachment surgery only 1 of the 90 patients developed the fishmouth phenomenon. The discrepancy between the expected incidence in the 2 series of patients and the actual incidence in the series of 90 patients may be accounted for as follows. It has been this author's practice to treat all primary detachments with superior tears, as defined, by means of an intravitreal air injection, cryopexy, and a no. 40 silicone tape, as previously described (Chawla and Birchall, 1973). Only patients with tears below the 4 o'clock and 8 o'clock meridia have had silicone-guttered explants placed securely beneath the no. 40 tape to buckle the sclera at the site(s) of tear(s). Thus more than $70 \%$ of these 90 patients have not had any scleral buckling performed. The 1 patient of this series of 90 who developed a fishmouth phenomenon was in the inferior tear group.

As a point of conjecture it may then be good prophylaxis to treat patients with large horseshoe tears primarily with intravitreal air or gas (Lincoff et al., 1977), cryopexy, and no scleral buckle. Other workers' results suggest this also (Chawla and Coleiro, 1977). The question arises, Why use a no. 40 silicone tape at all? It is thought that the use of such a tape with very minimal tension at or about the equator produces counteraction against any intravitreal retinal traction. As there may be vitreous traction at the rolled edges of large tears one has not yet been bold enough to discontinue its use. Such a method described above without the use of the tape may be the subject of a future pilot study.

The use of secondary cryopexy in previously frozen areas showing good chorioretinal reaction does not cause anxiety. Such secondary treatment does not preclude the production of new adhesive connections both intraretinally, between the rods and villi of the retinal pigment epithelial cells, and subretinally between the pigment epithelium and the collagen layers of Bruch's membrane and choroid. So long as the primary cryopexy does not cause total necrosis of the retinal pigment epithelium then the new cryoadhesions caused by the repeat 
cryopexy will be as strong as any initial cryoadhesions (Kreissig and Lincoff, 1975).

The use of intravitreal air in retinal detachment surgery is an old concept (Rosengren, 1938) which acquired a dubious reputation because of the occasional incidence of a massive intraocular infection. Modern surgical techniques appear to have eliminated this complication, and the intravitreal air method has now become accepted in many retinal units as part of their regular spectrum of surgical alternatives. Since the incidence of fishmouthing horseshoe tears may be predicted, this intravitreal air technique used in appropriate cases is a satisfactory method of virtually eliminating surgical failure due to the phenomenon. When fishmouthing has inadvertently occurred, the method is a safe and easy way in which to re-attach the retina, leaving the operated eye quiet within a few days of surgery.

\section{References}

Chawla, H. B., and Birchall, C. H. (1973). Intravitreal air in retinal detachment surgery. British Journal of Ophthalmology, 57, 60-70.

Chawla, H. B., and Coleiro, J. A. (1977). Retinal detachment treated with intravitreal air: An evaluation of 241 cases. British Journal of Ophthalmology, 61, 588-592.

Chignell, A. H. (1977). Retinal mobility and retinal detach- ment surgery. British Journal of Ophthalmology, 61, 446449.

Curtin, V. T. (1976). Clinical Ophthalmology, Vol. 5, Chapter 16, p. 23. Edited by T. Duane. Harper \& Row: New York. Kreiger, A. E., Hodgkinson, B. J., Frederick, A. R., and Smith, T. R. (1971). The results of retinal detachment surgery: Analysis of 268 operations with broad scleral buckle. Archives of Ophthalmology, 86, 385-394.

Kreissig, I., and Lincoff, H. A. (1975). Mechanisms of retinal attachment after cryosurgery. Transactions of the Ophthalmological Societies of the United Kingdom, 95, 148-157.

Leaver, P. K., and Cleary, P. E. (1975). Macular hole and retinal detachment. Transactions of the Ophthalmological Societies of the United Kingdom 95, 145-147.

Lincoff, H. A., Baras, I., McLean, J. (1965). Modifications to the Custodis procedure for retinal detachment. Archives of Ophthalmology, 73, 160-163.

Lincoff, H. A., Kreissig, I., Lafranco, F. (1977). Mechanisms of failure in the repair of large retinal tears. American Journal of Ophthalmology, 84, 501-507.

Pruett, R. C. (1977). The fishmouth phenomenon II. Archives of Ophthalmology, 95, 1782-1787.

Rosengren. B. (1938). Cases of retinal detachment treated with diathermy and injections of air into the vitreous body. Acta Ophthalmologica, 16, 177.

Schepens, C. L. (1965). Recent developments in the management of retinal detachment. Transactions of the American Academy of Ophthalmology and Otolaryngology, 69, 896911.

Shea, M., Maberley. A. L., and Weston, N. (1972). Two band procedure in retinal detachment surgery. Archives of Ophthalmology, 88, 388-393. 